\title{
Injusta Distribución del Presupuesto entre las Universidades Públicas del Ecuador: Efecto Mateo
}

\section{Unfair Distribution of the Budget Among the Public Universities of Ecuador: Matthew Effect}

\author{
Vicente Véliz Briones ${ }^{1}$, Juan Carlos Morales ${ }^{1}$, Gregorio Vásconez ${ }^{2}$ y Luz García Cruzatty ${ }^{1, *}$ \\ ${ }^{1}$ Universidad Técnica de Manabí, Ecuador \\ ${ }^{2}$ Universidad Técnica Estatal de Quevedo, Ecuador
}

\section{DESCRIPTORES:}

Presupuesto fisca

Universidad pública

Justicia social

Educación superior

Efecto Mateo

\begin{abstract}
RESUMEN:
En Ecuador, el sistema de educación superior ha experimentado una gran expansión tanto en número de estudiantes que aspiran obtener un título universitario como de instituciones públicas de educación superior (IPES). La mayoría de las cuales son financiadas por el estado, que históricamente ha asignado de forma imparcial los presupuestos beneficiando a IPES consideradas tradicionalmente elitistas, por su proceso de selección de estudiantes. El objetivo de la investigación fue establecer el impacto del efecto Mateo en IPES del Ecuador, durante el período 2014-2018. La metodología tuvo un diseño no experimental, descripto de desarrollo longitudinal. Un total de 26 IPES fueron analizadas en términos del presupuesto anual, eficiencia de ejecución, número de estudiantes, porcentaje destinado a la investigación y el número de publicaciones indizadas. Los datos fueron obtenidos de portales electrónicos y de la base de datos Scopus. Los resultados evidencian: 1) la mayor asignación presupuestaria a cuatro IES, sin relación al número de estudiantes o publicaciones, 2) los obstáculos financieros que afrontan las IES no elitistas para la consecución de sus planes de crecimiento, y 3 ) la necesidad de un cambio en esta injusta política fiscal, que no responde a principios de equidad y educación de calidad para todos.
\end{abstract}

\section{KEYWORDS:}

Fiscal budget

Public university

Social justice

Higher education

Mateo effect

\begin{abstract}
:
In Ecuador, higher education system has experienced a great expansion both in the number of students who aspire to obtain a university degree and in public institutions of higher education (PIHE). Most of which are funded by the government, which historically has been impartially allocated the budgets benefiting PIHE traditionally considered elitist, for its student selection process. The aim of this investigation was to determine the impact of the Mateo effect on PIHE from Ecuador, within the 2014-2018 period. The methodology used a non-experimental design to describe the longitudinal and transversal trends. Data of 26 PIHE was gathered from governmental web pages and the data base Scopus. The analysis took into consideration the annual budget, effectiveness of its execution, number of students, the percentage assigned for research as well as the number of publications indexed in Scopus. Overall results evidenced: 1) the greater budgets given to elite PIHE, regardless of their smaller number of students and indexed publications, 2) the financial constraints affronted by less elite PIHE that hindered their academic growth, and 3) the urgent need for a change in the prevailing financial politic, that counteracts the principles of equity and quality education for all ecuatorians.
\end{abstract}

CÓMO CITAR:

Véliz Briones, V., Morales, J. C., Vásconez, G. y García Cruzatty, L. (2021). Injusta distribución del presupuesto entre las universidades públicas del ecuador: Efecto Mateo. Revista Internacional de Educación para la Justicia Social, 10(1), 197-210. https://doi.org/10.15366/riejs2021.10.1.012

*Contacto: luz.garcia@utm.edu.edu.ec

ISSN: 2254-3139

revistas.uam.es/riejs
Recibido:

15 de agosto 2020

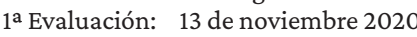

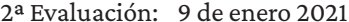

Aceptado: 16 de febrero 2021 


\section{Introducción}

Con la globalización, que anuncia la era de una economía basada en el conocimiento, las universidades juegan un rol primordial en la competitividad de los países (Lucchesi, 2011). Es por ello que fortalecer a las universidades se ha convertido en un tema trascendental a nivel mundial (Shin, 2009). En este contexto, es fundamental el aporte financiero estatal en el avance académico y científico de las universidades (Shibayama, 2011). Se espera que las estrategias de financiamiento estatal proporcionen incentivos directos para mejorar la formación profesional y la investigación científica, y así aportar positivamente a la economía de un país (Salter y Martin, 2001).

En América Latina, las instituciones públicas, por lo general, utilizan el modelo de asignación de recursos de "histórico-negociado", que se fundamenta en el incremento anual de presupuestos anteriores. Este modelo que predomina en la región, se basa en asignaciones presupuestarias por partidas específicas, y en algunos países también se utilizan mecanismos suplementarios como: recursos públicos adicionales; recursos de origen privado (pagos de matrícula por ejemplo), concesiones no reembolsables, venta de servicios; y obtención de recursos mediante cooperación internacional (García 2007). El modelo de "histórico-negociado", ha sido criticado por la falta de incentivos para incrementar la eficiencia y calidad, también por la ventaja de inicio que proporciona a determinadas instituciones (Amestica et al., 2014), constituyéndose con el tiempo en ventajas acumulativas producto del círculo vicioso ocasionado por el modelo.

En Ecuador, los sistemas de educación superior han experimentado un gran crecimiento tanto en número de estudiantes que aspiran obtener un título universitario, como de instituciones públicas de educación superior (IPES). Son estas instituciones financiadas por el gobierno las que ostentan la más diversa oferta académica, estando localizadas en las grandes ciudades, así como en cantones rurales, matriculando a un alto número de estudiantes, que en su mayoría son jóvenes de escasos recursos económicos (Espinoza, 2016). Sin embargo, es evidente que este aumento en la población estudiantil, no ha ido acompañada por un aumento proporcional en los presupuestos asignados por el Estado a las IPES.

Presupuestos deficitarios han comprometido el mantenimiento y modernización de las infraestructuras físicas y tecnológicas, incluyendo laboratorios de última generación, así como la instalación de aulas y bibliotecas virtuales y centros de excelencia académica. Presupuestos deficitarios redundan, también, en la disposición de IPES a contratar docentes/investigadores de alto nivel y afectan negativamente los programas de capacitación de los recursos humanos. Por otra parte, el presupuesto fiscal de todo Estado es finito y cada año es repartido entre las distintas entidades públicas dependiendo de las prioridades e importancia que el gobierno de turno asigne a cada entidad, mediado por la visibilidad de los actores solicitantes y de acuerdo a criterios socio-políticos que no necesariamente obedecen a planes estratégicos de desarrollo de las IPES y mucho menos a sus proyecciones presupuestarias solicitadas anualmente. Grupos específicos, de tradición histórica, pueden también ser la fuerza motriz detrás de la toma de decisiones sobre la dirección de las erogaciones presupuestarias por parte del Gobierno Nacional.

El "efecto Mateo" que tiene su origen en la Biblia, capítulo 13, versículo 12 del evangelio de San Mateo, denominada "parábola de los talentos dice: "Porque al que tiene se le dará y tendrá en abundancia; pero al que no tiene incluso lo que tiene se le quitará". Fue Robert K. Merton, reconocido sociólogo, a quién se le atribuye la aplicación del "efecto Mateo" al ámbito teórico y práctico de las ciencias, educación, sociopolítica, economía, y marketing (Jiménez-Rodríguez, 2009). Sin embargo, en el contexto de la educación superior en Ecuador, el "efecto Mateo" parecería también estar internalizado en los criterios para la asignación del presupuesto anual a las IPES, que se calcula utilizando una fórmula de distribución de recursos desde 2013 al 2018. Esta función real lineal de cuatro variables (calidad, excelencia, eficiencia administrativa y financiera), es un modelo multicriterio que considera indicadores relacionados con el sistema de evaluación institucional utilizado por El Consejo de Aseguramiento de la Calidad de la Educación Superior (CACES) (SENESCYT 2018).

Desafortunadamente, el presupuesto asignado durante estos años a las IPES ecuatorianas sugiere que la fórmula utilizada tiende a beneficiar a aquellas instituciones mejor posicionadas históricamente, limitando las oportunidades de otras IPES para mejorar y proporcionar una formación de 
calidad a los ecuatorianos. Así mismo, se ha ralentizado el trabajo de investigadores, quienes al carecer de recursos para sus investigaciones no han podido contribuir significativamente a solucionar los problemas de la sociedad ecuatoriana (Espinoza, 2016).

Estas políticas favorecen a las universidades que tienen mejores condiciones para competir, pues han fortalecido sus laboratorios, infraestructura, grupos de investigadores, logrando madurez institucional con el tiempo, y por lo cual acceden reiteradamente a recursos que facilitan la acumulación de beneficios. Por el mismo motivo, han conseguido tal prestigio que les facilita la captación de fondos externos para investigación y transferencia de tecnología. Es decir, un círculo vicioso provocado por una repartición inequitativa de los recursos estatales a través del tiempo, que indudablemente causan la exclusión a el acceso a fondos del resto de universidades (Amestica, 2014).

Bajo esta premisa, el presente trabajo tiene por objetivo caracterizar las implicaciones del "efecto Mateo" en 26 IPES del Ecuador, tomando como referencia el presupuesto asignado por el Estado y su relación con la producción científica, eficiencia administrativa, número de estudiantes y atención a la población vulnerable e históricamente excluida, durante el periodo 2014-2018.

\section{Método}

El estudio fue de tipo no experimental, descripto de desarrollo longitudinal, considerando 26 universidades públicas del Ecuador, con retrospectiva al periodo 2014-2018. Las IES públicas excluidas de este estudio corresponden a universidades de reciente creación, según decreto emitido por el gobierno nacional en el año 2013 (Yachay Tech, Universidad de las Artes, Universidad del Docente y Universidad Amazónica IKIAM). Para esto se evaluó el presupuesto general y de investigación (asignado y ejecutado), número de publicaciones Scopus, número de estudiantes (general y por etnias) en cada una de las IPES bajo análisis. Los datos fueron obtenidos a partir de portales web oficiales del Gobierno Ecuatoriano: Consejo de Participación Ciudadana y Control Social; Ministerio de Economía y Finanzas; Consejo de Evaluación, Acreditación y Aseguramiento de la Calidad de la Educación Superior y Secretaría de Educación Superior, Ciencia, Tecnología e Innovación. Los datos recopilados fueron sometidos a análisis de correlación y regresión entre: a) el presupuesto del año 2014 versus los presupuestos de los años 2015, 2016, 2017 y 2018; b) total de estudiantes inscritos versus presupuesto anual y c) el presupuesto para investigación asignado y el número de publicaciones en Scopus, base de datos que calcula factor de impacto y que es incluida en los criterios de evaluación por el Consejo de Aseguramiento de la Calidad de la Educación Superior en Ecuador (CACES). Además, se realizó un análisis de clúster para agrupar las 26 IPES en función del presupuesto anual y el presupuesto asignado para investigación. Con la agrupación de las IES, se realizó análisis de distribución de frecuencia de acuerdo a la asignación presupuestaria por estudiante y para investigación, inclusión de estudiantes por etnia y financiación de proyectos de investigación.

\section{Resultados}

Las relaciones entre el presupuesto del año 2014 con respecto al presupuesto de los años 2015, 2016, 2017 y 2018 (Figura 1) evidencian que la asignación del presupuesto a las IPES se ha mantenido estable de un año a otro dentro del periodo evaluado, ya que la pendiente (coeficiente b) fluctúa entre 0,98 y 1,11. Esto significa que aquellas IPES que recibieron un mayor presupuesto en el año 2014, también lo hicieron durante los siguientes 4 años, mientras que es evidente que las IPES que históricamente han recibido menores presupuestos siguieron recibiendo menos recursos. 
Figura 1

Correlación entre la asignación presupuestaria en el año 2014 y periodos 2015-2018

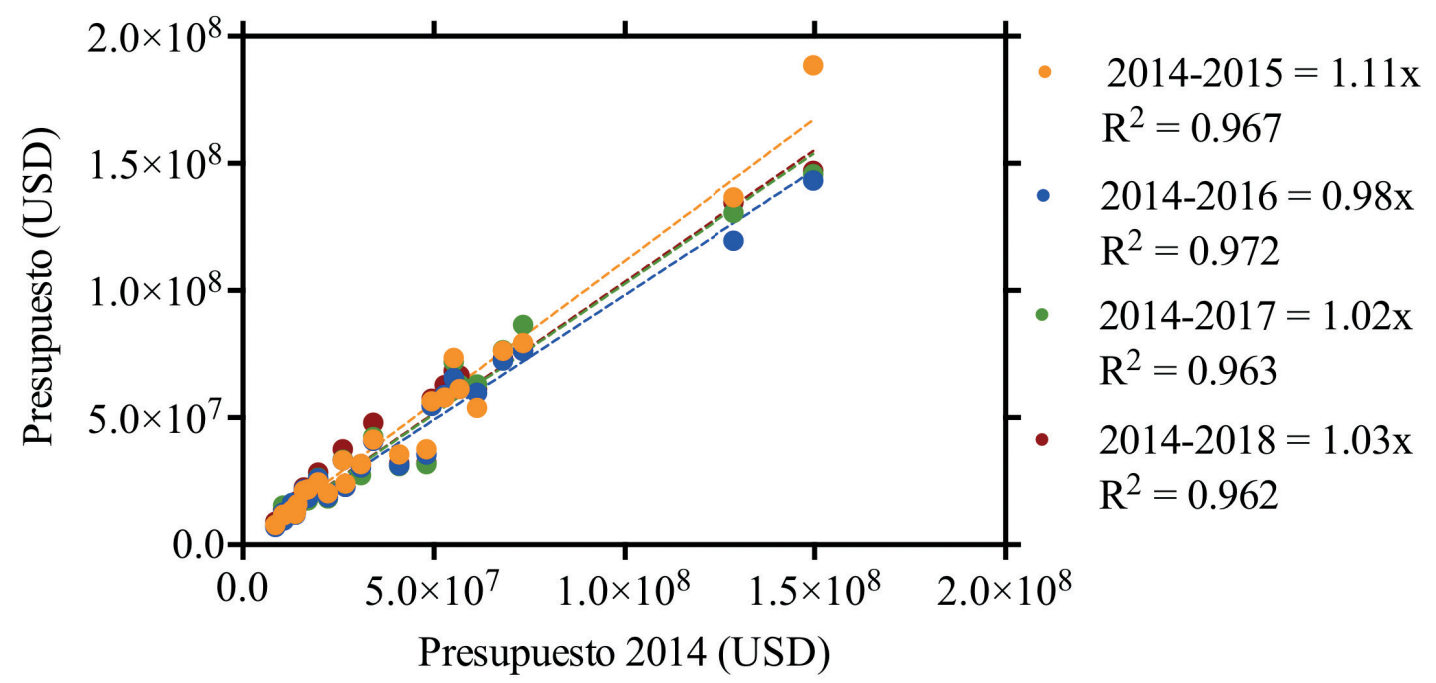

Por otra parte, se encontró una correlación positiva y significativa $2=0.597$ entre el número de estudiantes inscritos y el presupuesto anual de las IPES en los años 2014, 2015, 2016, 2017 y 2018 (Figura 2) indicando, a su vez, que el $40 \%$ de la variación observada en el presupuesto de las IPES responde a factores distintos al número de estudiantes inscritos.

Figura 2

Correlación entre el total de estudiantes y la asignación presupuestaria anual

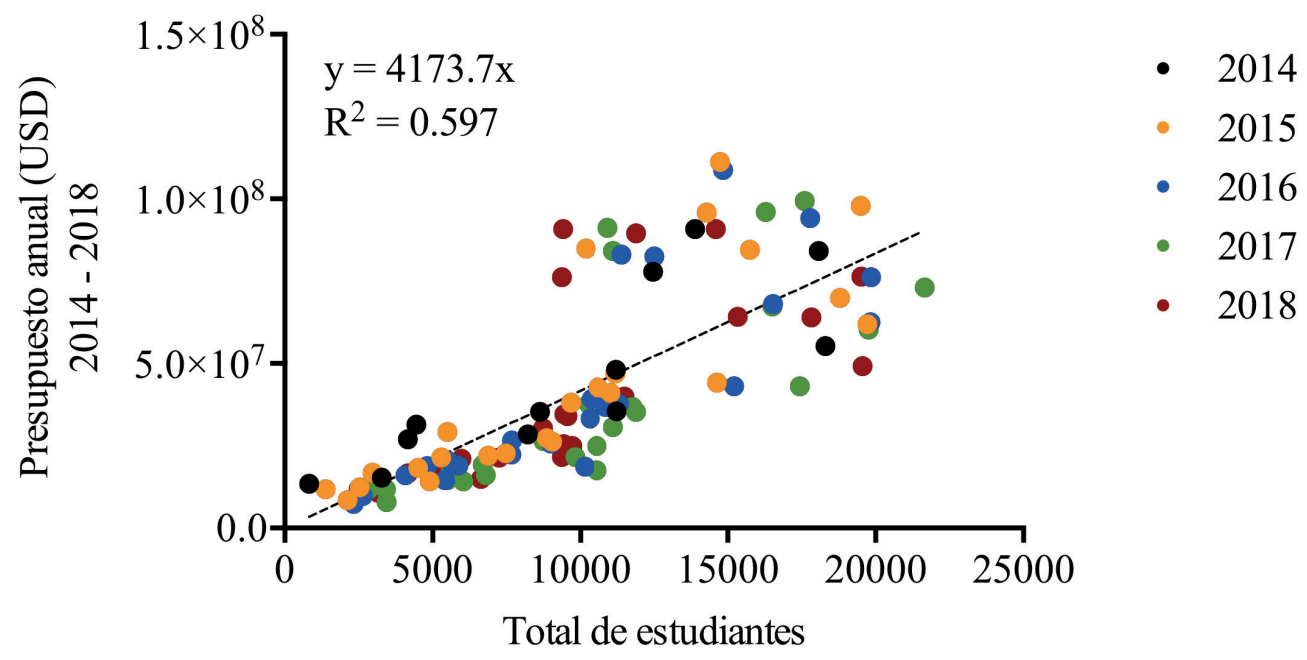

El análisis de clúster de acuerdo a la asignación presupuestaria por estudiante (Figura 4A) identifica claramente dos Grupos de IPES. El Grupo 1 conformado por 3 IPES: Universidad de las Fuerzas Armadas (ESPE), la Escuela Superior Politécnica del Litoral (ESPOL) y la Escuela Politécnica Nacional (EPN); y el Grupo 2 que congrega las otras 23 IEPS: Universidad de Cuenca (UC), Universidad Agraria del Ecuador (UAE), Universidad Técnica Luis Vargas Torres (ULVT), Universidad Estatal Península de Santa Elena (UPSE), Escuela Superior Politécnica del Chimborazo (ESPOCH), Universidad Técnica de Cotopaxi (UC), Universidad Estatal al Sur de Manabí (UNESUM), Universidad Estatal de Quevedo (UTEQ), Universidad Laica Eloy Alfaro de Manabí (ULEAM), Universidad Técnica de Babahoyo (UTB), Universidad Técnica de 
Manabí (UTM), Universidad Estatal de Milagro (UEM), Universidad Estatal de Bolívar (UB), Universidad Técnica del Norte (UTN), Universidad Técnica de Machala (UTM), Universidad Nacional de Chimborazo (UNACH), Universidad Politécnica Estatal del Carchi (UPEC), Universidad de Guayaquil (UG), Universidad Técnica de Ambato (UTA), Universidad Nacional de Loja (UNL), Universidad Central del Ecuador (UCE) y Escuela Superior Politécnica de Agropecuaria de Manabí (ESPAM).

\section{Figura 3}

Inequidad en el presupuesto asignado. Comparación entre las 3 IPES que tienen mayor asignación presupuestaria (Grupo 1: ESPE, ESPOL, EPN) y 3 IPES de similar población estudiantil (Grupo 2: UTM, ESPOCH, ULEAM).

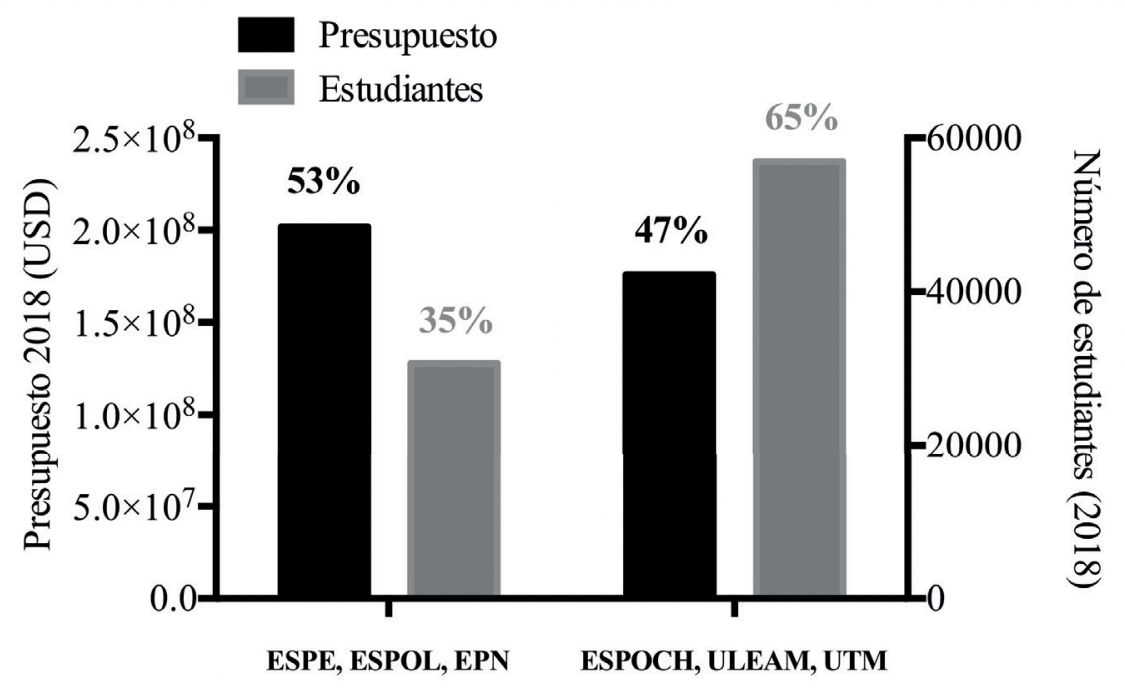

Esta fuerte disparidad entre el número de IPES consideradas Grupo 1 y las agrupadas en el Grupo 2 evidencia la gran inequidad que ha habido en la asignación del presupuesto, habiendo el Grupo 1 recibido de forma consecutiva a lo largo del período 2014-2018 presupuestos significativamente superiores al resto de las IPES del Grupo 2. Los valores publicados señalan una asignación por estudiante entre \$5.873 y 9.618 al Grupo 1, con un promedio por estudiante de 7.379 \$/año; mientras, que el presupuesto por estudiante asignado a las IPES del Grupo 2 se mantuvo en el rango de los $\$ 1.624$ a 6.976, con un presupuesto promedio por estudiante de 3.540 \$/año. En resumen, el presupuesto por estudiante del Grupo 1 fue en promedio 2,1 (210\%) veces más alto al presupuesto promedio asignado a las IES del Grupo 2 por el mismo concepto (Cuadro 1).

Cuadro 1

Asignación presupuestaria por estudiante en los periodos 2014-2018

\begin{tabular}{|c|c|c|c|c|c|c|c|c|}
\hline \multirow[b]{2}{*}{ Grupos } & \multirow[b]{2}{*}{$\mathbf{N}$} & \multirow{2}{*}{$\begin{array}{l}\text { Presupues- } \\
\text { to prome- } \\
\text { dio por } \\
\text { estudiante } \\
\text { (USD) }\end{array}$} & \multicolumn{6}{|c|}{ Población de estudiantes por año } \\
\hline & & & 2014 & 2015 & 2016 & 2017 & 2018 & $\begin{array}{c}\text { Prome- } \\
\text { dio }\end{array}$ \\
\hline ESPE, ESPOL, EPN & 3 & $\begin{array}{c}7379 \\
(5873- \\
9618)\end{array}$ & 36595 & 39261 & 38773 & 38353 & 30685 & 36733 \\
\hline Otras IES & 22 & $\begin{array}{c}3540 \\
(1624- \\
6976)\end{array}$ & 283009 & 294294 & 299795 & 320425 & 299126 & 299330 \\
\hline Total & 25 & & & & & & & 336063 \\
\hline
\end{tabular}

Nota: Datos tomados del Consejo de participación Ciudadana y Control Social (CPCCS), Ministerio de Economía y Finanzas (MEF), Consejo de Evaluación, Acreditación y Aseguramiento de la Calidad de la Educación Superior (CACES). Se excluyeron del análisis los datos de la Universidad Estatal Amazónica por considerarse atípicos 
Para dar una mejor idea de la inequidad en la distribución del presupuesto a las IPES graficamos los valores de presupuesto total y número de estudiantes del Grupo 1 y de tres IPES del Grupo 2, correspondiente al 2018 (Figura 3). En este punto hay que destacar que el sistema de educación superior ecuatoriano, durante el periodo 2014-2018, registró una población de 336.063 estudiantes, con el 11\% distribuido entre las IPES del Grupo 1, quedando el resto de los estudiantes (89\%) repartidos entre las IPES del Grupo 2 (Cuadro 1). Según el ejemplo del Cuadro 3, en una relación simplista de 1:1, sería de esperar que las tres IPES del Grupo 1 acogieran el 53\% del estudiantado en correspondencia con el presupuesto global que estas IPES reciben, lejos de eso reciben a un 35\% de estudiantes.

En paralelo, se pudo observar que el análisis de clúster en base al presupuesto asignado para la investigación, aglutinó a las IPES en los mismos Grupos 1 y 2 (Figura 4), con la única diferencia que, en este caso, la Universidad de Cuenca pasó a conformar el Grupo 1. Los valores obtenidos de la revisión de datos publicados nos permiten estimar que el presupuesto asignado para investigación a las universidades consideradas Grupo 1 osciló entre $\$ 2.500 .000$ y $\$ 16.200 .000$, con un promedio de 6.974 .606 \$año. Sin embargo, en el mismo periodo 2014 al 2018, el presupuesto del Grupo 2 destinado a investigación estuvo entre 6.200 a $\$ 5.200 .000$, con un promedio por IES de $990.000 \$ / a n ̃ o$. De estos valores se deduce que las IES del Grupo 1 recibieron, en promedio, 7 (705\%) veces más recursos para investigación que las IES categorizadas en el Grupo 2 (Cuadro 2).

Figura 4

Agrupamiento de las IES de acuerdo a la asignación presupuestaria por estudiante (A) y para investigación (B) (2014-2018)

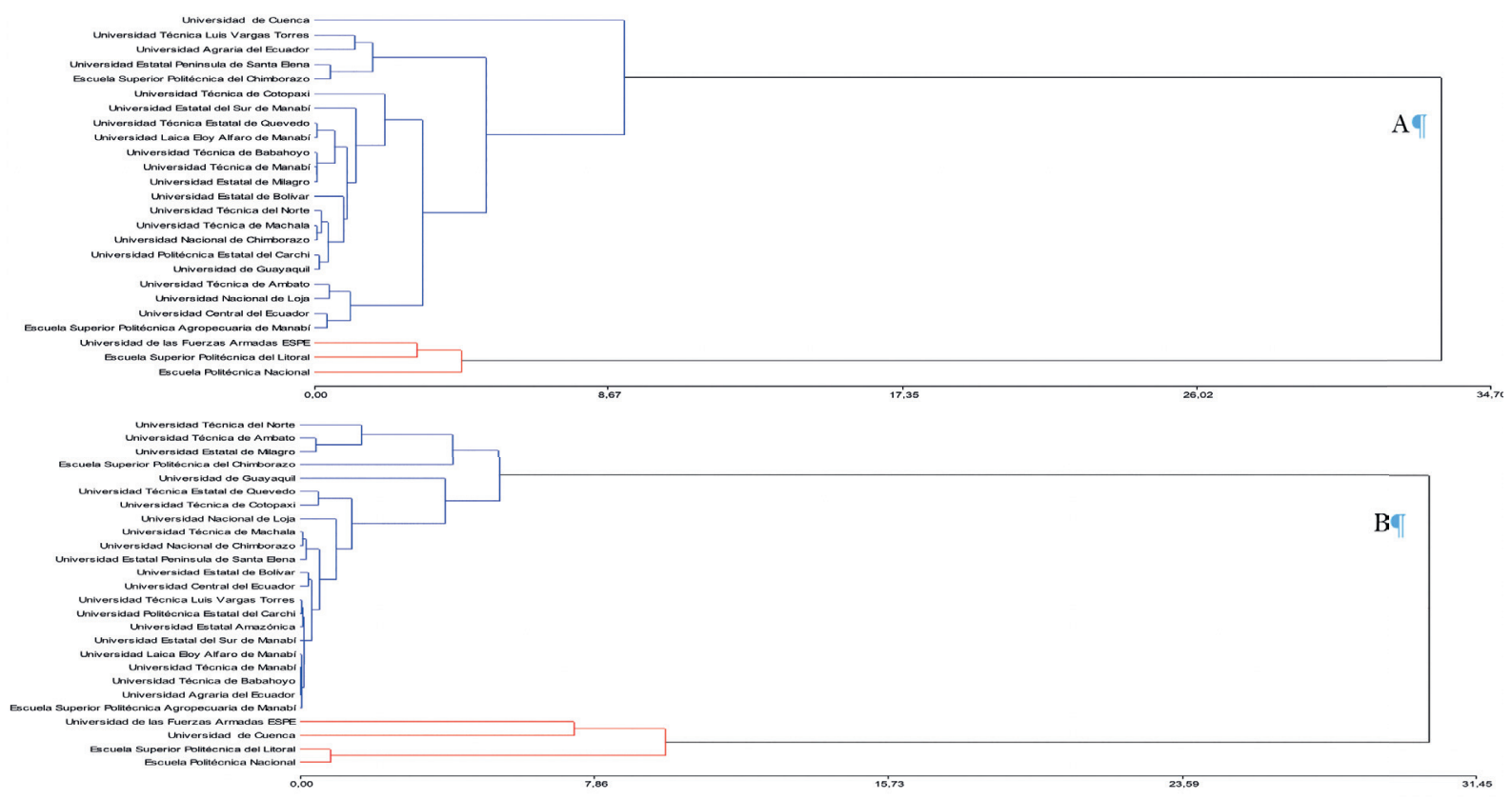

Con respecto al presupuesto para investigación, cabe indicar que los valores analizados corresponden al presupuesto asignado que no fue ejecutado en su totalidad. Durante el periodo de estudio, la efectividad promedio de ejecución del Grupo 1 fue del $75 \%$ del presupuesto planificado, mientras que las IES del Grupo 2 en el mismo período tuvieron un promedio de $88 \%$ de eficiencia en la ejecución de las respectivas partidas presupuestarias (Cuadro 2, Figura 5). 
Cuadro 2

Asignación presupuestaria para investigación en los periodos 2014-2018

\begin{tabular}{|c|c|c|c|c|c|c|c|c|c|}
\hline \multirow{2}{*}{ Grupos } & \multirow{2}{*}{$\mathbf{n}$} & \multirow{2}{*}{$\begin{array}{c}\text { Presupuesto } \\
\text { promedio para } \\
\text { investigación por } \\
\text { IES (USD) }\end{array}$} & \multicolumn{5}{|c|}{ Presupuesto promedio anual (USD) } & \multirow{2}{*}{$\begin{array}{c}\text { Presupuesto } \\
\text { total IES (USD) } \\
(2014-2018)\end{array}$} & \multirow{2}{*}{$\begin{array}{c}\text { Publica- } \\
\text { ciones en } \\
\text { Scopus }\end{array}$} \\
\hline & & & 2014 & 2015 & 2016 & 2017 & 2018 & & \\
\hline EPN, ES- & & 6974606 & & & & & & & \\
\hline $\begin{array}{l}\text { POL, ESPE, } \\
\text { UC }\end{array}$ & 4 & $\begin{array}{l}(2500000- \\
16200000)\end{array}$ & 6532626 & 4736065 & 7354738 & 10265615 & 5983983 & 139492110 & 3999 \\
\hline Otras IES & 22 & $\begin{array}{c}990000 \\
(6200-5200000)\end{array}$ & 881019 & 1015969 & 1129674 & 922252 & 1001085 & 108899997 & 4342 \\
\hline Total & 26 & & & & & & & 248392107 & 8341 \\
\hline
\end{tabular}

Nota: Elaboración propia. Datos tomados del Consejo de participación Ciudadana y Control Social (CPCCS), Ministerio de Economía y Finanzas (MEF), Scopus 17-05-19.

Figura 5

Eficiencia en ejecución presupuestaria, presupuesto y población de estudiantes (2014-2018)



Figura 6

Eficiencia y porcentaje del presupuesto asignado a la investigación en comparación con el número de publicaciones citadas en la base de datos Scopus

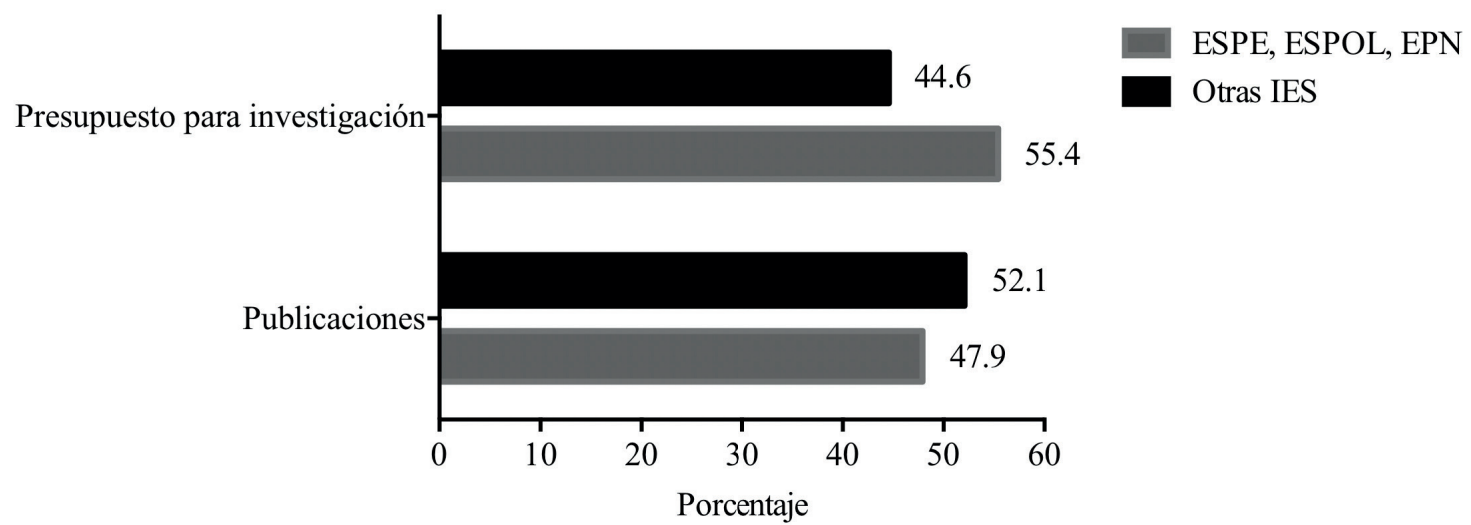


El número de publicaciones en revistas indexadas es un indicador universal de la efectividad de las investigaciones en generar nuevos conocimientos. Los datos recopilados correspondientes al periodo 2014-2018 revelan un total de 8.341 artículos publicados en revistas indexadas en Scopus (Cuadro 2). Como indicamos anteriormente, el Estado durante el periodo 2014-2018 destinó 55,4 \% del presupuesto para investigación a tres instituciones, las IPES del Grupo 1 y 44,6\% restante al Grupo 2 . Sin embargo, sólo el $48 \%$ de esas publicaciones fueron generadas por el Grupo 1, mientras el 52 \% es de autoría de profesores del Grupo 2 (Figura 5). En una relación simplista de 1:1, sería de esperar que las cuatro IPES del Grupo 1 publicaran al menos el $55,4 \%$ del total en correspondencia con el presupuesto que reciben para investigación.

Figura 7

Presupuesto promedio para investigación, promedio de artículos en Scopus (2014-2018) y costos de artículos

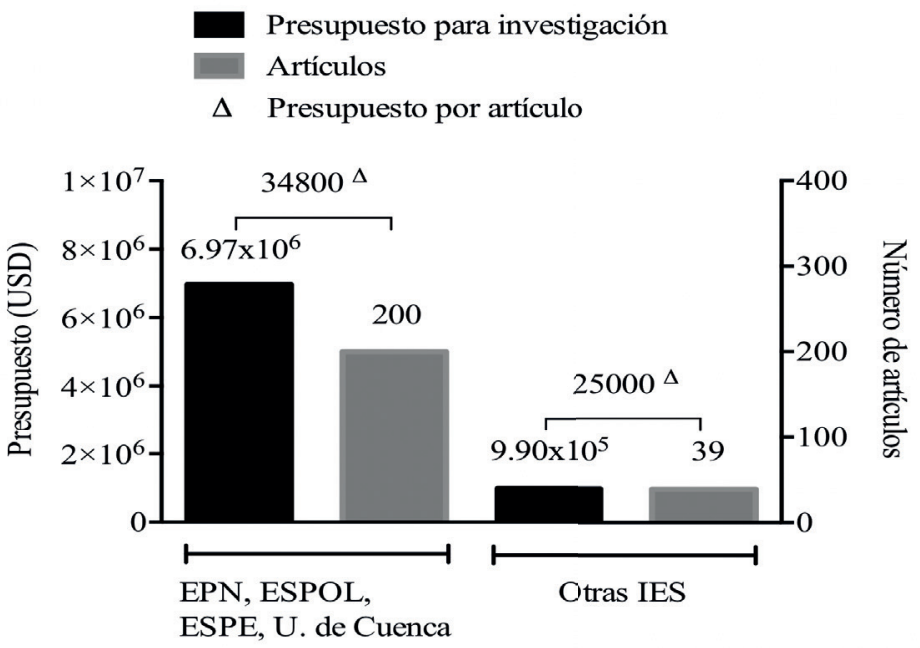

Según los datos, el Grupo 1 publicó un promedio de 200 artículos/año en revistas indexadas en Scopus, con un costo promedio de 34.800 \$/artículo. Por su lado, el Grupo 2 publicó un promedio de 39 artículos a un costo cada de 25.000 \$ (Figuras 6 y 7). Según los datos analizados, no existe una relación estadística entre el número de publicaciones y el presupuesto asignado para investigación (Figura 8).

Es importante destacar, que además del presupuesto asignado a estas IES, la Secretaría Nacional de Educación Superior, Ciencia y Tecnología (SENESCYT) financió, durante el periodo en estudio, numerosos proyectos de investigación a las IES del Grupo 1, un total del 75,6\% de los proyectos aprobados fueron para estas instituciones (Cuadro 3).

Figura 8

Correlación entre presupuesto asignado a investigación y publicaciones Scopus




Cuadro 3

Proyectos financiados por Senescyt a Instituciones de Educación Superior Públicas 2014-2018

\begin{tabular}{|c|c|c|c|c|c|c|c|c|c|c|}
\hline & $05-06$ & 08 & 11 & 13 & 14 & 15 & 16 & 17 & 18 & Total \\
\hline $\begin{array}{l}\text { Escuela Superior Politécnica del } \\
\text { Litoral - ESPOL }\end{array}$ & 6 & 2 & 9 & - & 6 & 1 & - & 1 & 9 & 34 \\
\hline $\begin{array}{l}\text { Escuela Politécnica Nacional } \\
\text { (EPN) }\end{array}$ & 13 & 7 & 4 & - & 1 & 2 & - & - & 3 & 30 \\
\hline $\begin{array}{l}\text { Universidad de las Fuerzas Ar- } \\
\text { madas (ESPE) }\end{array}$ & 1 & 2 & 2 & - & 1 & 2 & - & 1 & 5 & 14 \\
\hline Universidad de Cuenca (UC) & 2 & - & 1 & 5 & 1 & 2 & - & - & 1 & 12 \\
\hline $\begin{array}{l}\text { Escuela Superior Politécnica de } \\
\text { Chimborazo (ESPOCH) }\end{array}$ & 8 & - & - & - & 1 & - & - & - & - & 9 \\
\hline $\begin{array}{l}\text { Universidad Central del Ecuador } \\
\text { (UCE) }\end{array}$ & 4 & 1 & - & - & 1 & - & - & - & 1 & 7 \\
\hline $\begin{array}{l}\text { Universidad Nacional de Loja } \\
\text { (UNL) }\end{array}$ & 1 & 3 & 2 & - & - & - & - & - & - & 6 \\
\hline $\begin{array}{l}\text { Universidad Técnica Estatal de } \\
\text { Quevedo (UTEQ) }\end{array}$ & 1 & 2 & - & - & - & - & - & - & - & 3 \\
\hline $\begin{array}{l}\text { Universidad Técnica de Manabí } \\
\text { (UTM) }\end{array}$ & 1 & 1 & - & - & - & - & - & - & 1 & 3 \\
\hline $\begin{array}{l}\text { Universidad Técnica de Ambato } \\
\text { (UTA) }\end{array}$ & - & - & - & - & - & - & - & 1 & 2 & 3 \\
\hline Universidad Técnica de Cotopaxi & 1 & - & - & - & - & - & - & - & - & 1 \\
\hline $\begin{array}{l}\text { Universidad Técnica Luis Vargas } \\
\text { Torres de Esmeraldas }\end{array}$ & 1 & - & - & - & - & - & - & - & - & 1 \\
\hline $\begin{array}{l}\text { Universidad Estatal de Milagro } \\
\text { (UNEMI) }\end{array}$ & - & - & - & - & - & - & - & - & 1 & 1 \\
\hline
\end{tabular}

Nota: Datos tomados de la página de la Secretaría de Educación Superior, Ciencia, Tecnología e Innovación (SENESCYT).

Finalmente, se revisaron los datos disponibles del 2018 sobre la etnia de los estudiantes matriculados en las IPES en Ecuador. Del total de 29.723 estudiantes matriculados que se identifican con alguno de los grupos históricamente excluidos, solo el 6,3\% ingresó a las IPES clasificadas en el Grupo 1. De este total de estudiantes, el 40,8\% se auto identificó como montubio y sólo el 1\% ingresó a alguna de las 3 IPES del Grupo 1 (Figura 9).

Figura 9

Total de estudiantes en Grupos históricamente excluidos. Comparación entre Universidades que reciben mayor asignación presupuestaria por estudiante y el resto de las IES, año 2018

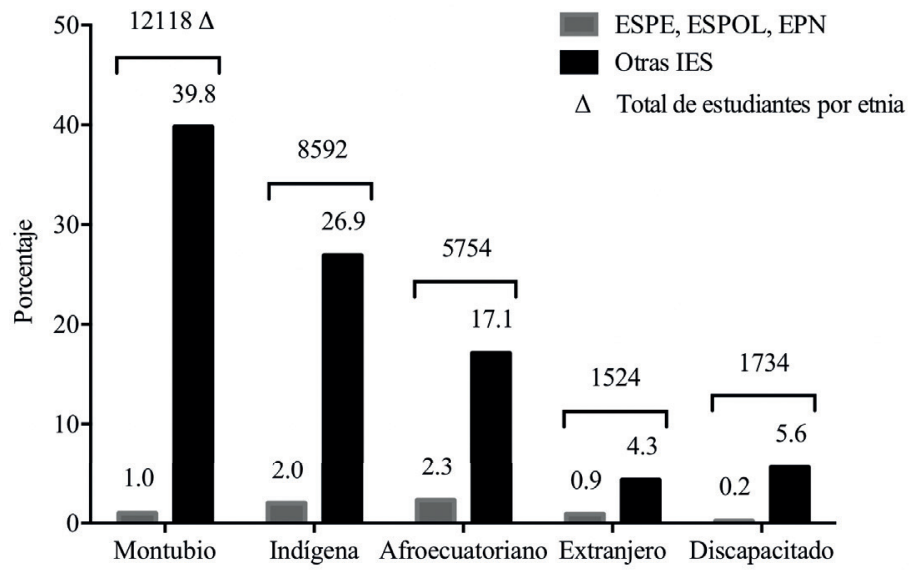




\section{Discusión}

En Ecuador, la obligación del Estado de asegurar la educación superior a todos sus habitantes, con iguales oportunidades para acceder a la máxima calidad y pertinencia de la enseñanza, está claramente establecida en los artículos 29 y 52 de la Constitución de la República. Por otra parte, la Ley Orgánica de Educación Superior (LOES), en su Artículo 3, señala el derecho de todos a recibir una formación superior de carácter humanista, cultural, científica y tecnológica que responda al interés público, no a intereses individuales. En el marco de estos mandatos, cada IPES ha diseñado un conjunto de estrategias para garantizar el nivel académico y de investigación, siempre con la excelencia como objetivo final. Sin embargo, los resultados de esta investigación evidencian la inequidad en la asignación de recursos a las Instituciones de Educación Pública del Ecuador. Se ha evidenciado la internalización del "efecto Mateo" en la distribución de los presupuestos a las IPES ecuatorianas ya que, de forma recurrente, a un grupo de instituciones se les ha acreditado los presupuestos más altos, en menoscabo del resto de instituciones.

Los resultados de este trabajo apuntan, además, hacia la poca importancia que se otorga a los criterios de número de estudiantes matriculados, situación socio-económica de las provincias de adscripción y el nivel de las investigaciones llevadas a cabo de impacto directo a la sociedad, a la hora de repartir el presupuesto nacional entre las IPES que constituyen la matriz de la educación superior en Ecuador. Más aún, todas las IPES, de manera injusta, desde el 2013 son evaluadas con el mismo baremo de productividad científica, sin reparo en el monto del presupuesto para ello otorgado y del número de estudiantes matriculados. En otras palabras, todas las IPES en Ecuador son injustamente medidas con la misma vara, con resultados negativos para la mayoría. En un "efecto boomerang", la menor productividad de las IPES a las que se asigna un menor presupuesto (Grupo 2) convence a los burócratas administrativos del Estado de asignarles el mismo o menor presupuesto que el año anterior, con la acumulación de ventajas para las Instituciones con mayor presupuesto.

La mayoría de estas IPES carecen de infraestructuras de investigación debido al déficit presupuestario que confrontan cada año. Sin laboratorios con tecnologías de punta y bibliotecas con conexión gratis on line a las más importantes bases de datos sociales y científicas, es básicamente imposible exigirles a los docentes/investigadores generar nuevos conocimientos de alto impacto. Por otra parte, la presión ejercida por la misma sociedad para que IPES del Grupo 2 incrementen de manera sistemática anual el cupo para estudiantes debería ir acompañado por un aumento substancial en los presupuestos acreditados. Una mayor población estudiantil requiere de nuevas infraestructuras, y va de la mano de un aumento en la nómina de docentes.

Estas universidades, pese a sus esfuerzos, precisarán mucho tiempo para conseguir el nivel de excelencia que han logrado las instituciones de mayor presupuesto. Es evidente que, al igual que en otros países como Chile "existe una deuda histórica con estas universidades, y cuya reivindicación es necesaria si se quiere tener un país más justo e igualitario. Lo anterior no supone una "estrategia tipo Robin Hood", es decir, quitarle a lo más ricos para darle a los pobres, sino más bien reconocer esta brecha y fortalecer a las más desventajadas” (Amestica et al., 2014).

Mucho antes de la nueva constitución, Estrella (2011) planteó la ausencia en Ecuador de una fórmula ideal que vincule tanto el número actual de estudiantes como su incremento en el tiempo con las asignaciones presupuestarias a las IPES. Por otra parte, Amestica y otros (2014) y Tarango y Machín-Mastromatteo (2016) sostienen que se debe reconocer la deuda histórica y definir el mecanismo de reivindicación del Estado en procura de reducir la brecha presupuestaria entre IPES, con el fin de lograr una educación superior más justa e igualitaria. Según David (1994), este efecto tangible del financiamiento pasado y reciente se explica cómo la dinámica de ventajas acumulativas, indicando que solo los equipos más hábiles en conseguir financiamientos disponen de mayores recursos para sus investigaciones. El informe de la SENESCYT (2018) concluye que solo dos IPES recibieron el $48.01 \%$ en el año 2017, y el $47.17 \%$ en el año 2018 del presupuesto asignado al criterio de excelencia en investigación, según la fórmula utilizada para la asignación de recursos a las IPES en Ecuador. En nuestra opinión, existe una redundancia entre los criterios de calidad y excelencia, pues los tres indicadores se evalúan bajo los mismos términos de gasto en investigación y el desarrollo, producción científica-regional. Por ejemplo, 
este informe señala que la Escuela Politécnica del Litoral (ESPOL) recibió cerca de 14 millones de dólares por concepto de estos tres indicadores redundantes.

Según (Escobar 2016), esta ventaja acumulativa en la educación superior también se refleja en la adjudicación de becas para estudiar postgrados, pues justamente las IPES a las que se ha adjudicado mayor presupuesto (2014-2018) están entre las IPES con más graduados que han obtenido becas de la Secretaría Nacional de Ciencia y Tecnología del Ecuador (2009-2014). Estos datos demuestran que "los procedimientos aplicados siguen perpetuando los niveles de exclusividad de acceso, compensando a los ya compensados, provocando algo similar al "efecto Mateo", descrito por Merton, (1968), Espinoza, (2013) y Esper y otros (2015). Sin embargo, la asignación histórica de un mayor presupuesto a un pequeño grupo de universidades evidencia una absoluta ineficiencia en la distribución de los recursos, lo que genera limitaciones en la solución de problemas de la sociedad, como lo señala Nieto y otros (2015).

Por otra parte, cabe mencionar que las IPES del Grupo 2, siempre menos favorecidas en términos de presupuesto, son las que muestran mejores valores de equidad e inclusión social, brindando el apoyo a las etnias históricamente excluidas del sistema de instrucción universitaria. Este punto contradice los principios Constitucionales sobre la igualdad de oportunidades para el ingreso de todos los ecuatorianos al sistema de educación superior (Van Hoof et al., 2013). Son las IPES del Grupo 2 las que enfrentan el reto de atender las múltiples diferencias de identidades étnicas que requiere la interculturalidad, a través de un diálogo más cercano entre la comunidad universitaria y las poblaciones étnicas (Londoño, 2017), y mediante políticas institucionales que aseguren la matrícula a estudiantes con mayor vulnerabilidad social (Gaete, 2015) y (Jiménez et al., 2017). Estos problemas de inequidad, no son exclusivos del Ecuador, más bien, se ha evidenciado altos niveles de segregación por nivel socioeconómico en la región (Carrillo, 2020).

\section{Conclusiones}

Basándonos en los resultados de este análisis podemos concluir que la fórmula actual utilizada por el Estado para calcular la asignación del presupuesto a cada IPES demuestra un sesgo o biass hacia aquellas IPES del Grupo 1 que históricamente han recibido mayores aportes económicos; además, evidenciadas en este estudio como deficientes ejecutoras presupuestarias. Es muy interesante notar que estas IPES elitistas, a pesar de contar con mayores presupuestos e infraestructuras instaladas de investigación, la magnitud del presupuesto recibido no es proporcional al número de estudiantes, ni a la producción científica, además presentan bajos niveles de inclusión a población vulnerable y un menor nivel de eficiencia administrativa en la ejecución de su presupuesto. Estas observaciones comprueban la presencia del "efecto Mateo" en el comportamiento injusto y discriminativo del Estado con respecto a las IPES del Grupo 2, principalmente aquellas localizadas en provincias periféricas, violando así los principios Constitucionales de equidad, igualdad e inclusión. Presupuestos deficitarios son, por lo tanto, el obstáculo principal que confrontan las IPES menos elitistas para el desarrollo de sus planes de crecimiento estudiantil, infraestructuras e instalación de laboratorios de docencia e investigación de alto nivel.

El presente análisis tiene importantes implicaciones en la implementación de nuevas políticas de financiamiento para las IPES del Grupo 2 sometidas a la tremenda presión ejercida por el Estado para aumentar el número de cupos de estudiantes por carrera y la apertura de nuevas carreras de pre y postgrado. Sin embargo, el "efecto Mateo" pone en relieve la necesidad de una base de datos coherente y centralizada que permita proyectar el costo de la expansión de la educación superior con sus retos tecnológicos en los años venideros. El cálculo del presupuesto con sus planes de ejecución y necesidades para el año siguiente son de obligatoria presentación por parte de las IPES. Sin embargo, estos documentos son raramente tomados en cuenta por parte del Estado.

\section{Sugerencias}

Basados en las conclusiones anteriores y habiendo demostrado la internalización del "efecto Mateo" en el cálculo del presupuesto para las IPES, proponemos al Estado ecuatoriano: 1. la reformulación de los procesos administrativos que determinan los presupuestos, eliminando las injusticias antes demostradas y permitiendo a IPES del Grupo II, con historial de bajo financiamiento, el acceso a mayores 
presupuestos; 2. permitir a las IPES del Grupo 2 la opción de solicitar créditos adicionales para cubrir deficiencias administrativas en caso, por ejemplo, de desastres naturales como el terremoto en Manabí en el 2016, imprevistos tales como la aparición de una epidemia (en humanos, animales o cultivos) que requiera de una inmediata investigación, o modernización del equipamiento para docencia; 3 . la asignación del presupuesto considerando nuevos parámetros como son: (i) el número de estudiantes registrados y su incremento en el futuro inmediato estimado por modelos poblacionales; y (ii) el impacto de las investigaciones reflejado en publicaciones en revistas indizadas en importantes bases de datos como son: Scopus, Web of Science, Science Citation Index, entre otras; así como un reconocimiento de la relevancia de los índices "h y hi10" como indicadores del nivel de las investigaciones publicadas.

Los resultados de todos los análisis aquí presentados coinciden en la imperiosa necesidad de eliminar el "efecto Mateo" de la distribución presupuestaria de las IPES en Ecuador.

\section{Referencias}

Amestica, L., Gaete, H. y Llinas-audet, X. (2014). Segmentación y clasificación de las universidades en Chile: desventajas de inicio y efectos de las políticas públicas de financiamiento. Ingeniare. Revista Chilena de Ingeniería, 22(3), 384-397. https://doi.org/10.4067/S0718-33052014000300009

Carrillo, S. (2020). La segregación escolar en América Latina. ¿Qué se estudia y cómo se investiga? REICE. Revista Iberoamericana sobre Calidad, Eficacia y Cambio en Educación, 18(4), 345-362. https://doi.org/10.15366/reice2020.18.4.014

David, P. (1994), Positive feedback and research productivity in science: Reopening another blackbox. En O. Grandstrand (Ed.), Economics of technology (pp. 54-89). Elsevier.

Escobar, C. (2016). Análisis de la política ecuatoriana de becas de estudios de posgrado en el exterior y su relación con el cambio de matriz productiva. Revista Latinoamericana de Políticas y Acción Pública, 3(2), 23-49. https://doi.org/10.17141/mundosplurales.2.2016.2842

Esper, R., Pérez, A. y Carrillo, L. (2015). San Mateo, la Malinche y las publicaciones científicas mexicanas. Revista de la Asociación Mexicana de Medicina, 29(3),128-132. http://www.scielo.org.mx/scielo.php?script=sci_arttext\&pid=S0187-84332015000300001\&lng=es

Espinoza, O. (2013). Equidad e inclusividad en la educación superior en los países andinos: Los casos de Bolivia, Chile, Colombia y Perú. Ediciones Universidad UNINF.

Espinoza, C. (2016). Calidad de la educación e índices de gestión en relación con el presupuesto de las universidades del Ecuador en el año 2015. Revista Universidad y Sociedad, 8(2).

Estrella, M. (2011). Free public universities in Ecuador: Too much of a good thing? International Higher Education, 65, 22-24. https://doi.org/10.6017/ihe.2011.65.8576

Gaete, R. (2015). Responsabilidad social en el gobierno y gestión de las universidades estatales chilenas. Civilizar, 15(29), 163-180.

García, C. (2007). Financiamiento de la educación superior en América Latina. Sociologías, 9(17), 50-101. https://doi.org/10.1590/S1517-45222007000100004

Jiménez, F., Lalueza, J. y Fardella, C. (2017). Aprendizajes, inclusión y justicia social en entornos educativos multiculturales. Revista Electrónica de Investigación Educativa, 19(3), 10-23. https://doi.org/10.24320/redie.2017.19.3.830

Jiménez-Rodríguez, J. (2009). El efecto Mateo: Un concepto psicológico. Papeles del Psicólogo, 30(2), 145-154.

Londoño, S. (2017). Estudiantes indígenas en universidades en el suroccidente colombiano: Tensiones entre calidad y pertinencia. Perfiles Educativos, 39(157), 52-69. https://doi.org/10.22201/iisue.24486167e.2017.157.58440

Lucchesi, M. (2011). La universidad internacional en América Latina: Un nuevo paradigma para el siglo XXI. Formación Universitaria, 4(1), 25-36. https://doi.org/10.4067/S0718-50062011000100005

Merton, R. (1968). The Matthew Effect in Science. Science, 159(3810), 56-63.

https://doi.org/10.1126/science.159.3810.56 
Nieto, L., Pérez, C., y Gómez, J. (2015). Financiación y Eficiencia en las Universidades Públicas Españolas. Investigaciones de Economía de la Educación, 10(9), 509-520.

Salter, A.J. y Martin, B.R., (2001). The economic benefits of publicly funded basic research: a critical review. Research Policy, 30(3), 509-532. https://doi.org/10.1016/S0048-7333(00)00091-3

República del Ecuador. (2008). Constitución de la República del Ecuador. Registro Oficial.

República del Ecuador. (2010). Ley Orgánica de Educación Superior. Registro Oficial.

SENESCYT. (2018). Informe sobre la metodología de distribución de recursos destinados anualmente por parte del Estado a favor de las instituciones de educación superior. SENESCYT.

Shibayama, S. (2011). Distribution of academic research funds: A case of Japanese national research grant. Scientometrics, 88(1), 43-60. https://doi.org/10.1007/s11192-011-0392-z

Shin, J.C. (2009). Building world-class research university: The Brain Korea 21 project. Higher Education, 58(5), 669 688. https://doi.org/10.1007/s10734-009-9219-8

Tarango, J. y Machín-Mastromatteo, J. (2016). Scientific production in Mexican universities: Rates and expectations toward competitiveness. Information Development, 32(1), 107-111. https://doi.org/10.1177/0266666915613730

Van Hoof H., Estrella M., Eljuri M. y Torres L. (2013). Ecuador's higher education system in times of change. Journal of Hispanic Higher Education 12(4), 345-355. https://doi.org/10.1177/1538192713495060

\section{Breve CV de los/as autores/as}

\section{Vicente Véliz Briones}

Doctor en Ciencias Técnicas por la Universidad Tecnológica José Antonio Echeverría de Cuba, Máster en Informática, Rector de la Universidad Técnica de Manabí durante los años 2013 a 2017 y reelegido en 2017 para otro periodo de 4 años. Durante su trayectoria académica ha sido decano de la Facultad de Ciencias Informáticas y Profesor Principal en la misma Facultad. También se ha desempeñado en varios cargos de dirección en el sector público. Como investigador, ha realizado varias publicaciones en el ámbito de la Gestión Universitaria, Educación e Informática. Email: vicente.veliz@utm.edu.ec

ORCID ID: https://orcid.org/0000-0003-4092-1421

\section{Juan Carlos Morales}

Doctor en Educación por la Universidad Nacional Mayor de San Marcos, Máster en Gerencia Educativa. Ingeniero Comercial. Decano de la Facultad de Ciencias Humanísticas y Sociales en la Universidad Técnica de Manabí (Portoviejo, Manabí, Ecuador. Código: 13015) y Profesor Principal en la misma Facudtad. Editor Técnico de la Revista de Ciencias Humanísticas y Sociales REHUSO ISSN 2550-6587. Investigador Acreditado SENESCYT. Áreas de investigación: e-Learning, Estudios Métricos de la Información, Alfabetización Informacional. Email: juan.morales@utm.edu.ec

ORCID ID: https://orcid.org/0000-0003-1021-8735

\section{Gregorio Vásconez}

Doctor en Ciencias Agrarias por la Universidad Austral de Chile, Máster en Nutrición Vegetal por la Universidad Tecnológica Equinoccial (Ecuador), Ingeniero Agropecuario. Asistente de investigación del Área Agrícola de la Universidad Técnica Estatal de Quevedo (UTEQ) del 2008 al 2015. Profesor Agregado de la UTEQ a cargo de las asignaturas Química de Suelos y Nutrición Vegetal. Director de la Revista Ciencia y Tecnología (ISSN 1390-4051; e-ISSN 1390-4043): https://revistas.uteq.edu.ec/index.php/cyt. Email: gvasconez@uteq.edu.ec

ORCID ID: https://orcid.org/0000-0003-1260-8075 


\section{Luz García Cruzatty}

Doctora en Ciencias Forestales por la Universidad Austral de Chile, Ingeniera Forestal. Investigadora acreditada por la Secretaría Nacional de Educación Superior, Ciencia y Tecnología (SENESCYT), actualmente es Directora del Instituto de Investigación de la Universidad Técnica de Manabí (Portoviejo, Manabí, Ecuador. Código: 13015) y Profesora Principal de la Facultad de Ingeniería Agronómica (FIAG) en la misma Universidad. Áreas de Investigación: Mejoramiento Genético, Biología Reproductiva de Plantas, y Gestión Universitaria. Email: luz.garcia@utm.edu.ec

ORCID ID: https://orcid.org/0000-0003-2625-7472 